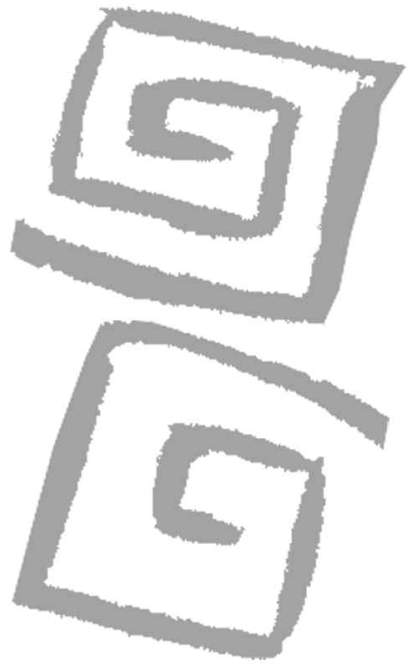

${ }^{1}$ Doctor en Ciencias Biológicas. Director, Instituto Nacional de Medicina Tropical. Investigador CONICET, Argentina. odanielsalomon@gmail.com

${ }^{2}$ Doctora en Antropología Social. Investigadora CONICET, Argentina. andreaveronicamastrangelo@gmail.com

${ }^{3}$ Doctora en Ciencias Naturales. Investigadora Asistente, Centro Nacional de Diagnóstico e Investigación de Endemoepidemias, ANLIS, Argentina.

mariasoledadsantini@gmail.com

${ }^{4}$ Magister en Efectividad Clínica. Médica asistente, Hospital de Pediatría Dr. Juan P. Garrahan, Argentina. sruvinsky@hotmail.com

${ }^{5}$ Médico Infectólogo. Jefe del Servicio, CEMPRA-MT, Hospital de Infecciosas

F. J. Muñiz, Argentina.

torduna@intramed.net

${ }^{6}$ Médico Veterinario. Jefe Servicio Patología, Instituto Nacional de Parasitología Dr. Mario Fatala Chabén, ANLIS, Argentina.

ajsinagra@yahoo.com.ar

${ }^{7}$ Médica Veterinaria, Instituto Nacional de Parasitología Dr. Mario Fatala Chabén, ANLIS, Argentina. caluna00@yahoo.com

${ }^{8}$ Médica. Jefa de Departamento Clínica, Patología y Tratamiento, Instituto Nacional de Parasitología Dr. Mario Fatala Chabén, ANLIS, Argentina.ariarte@yahoo.com

${ }_{9}$ Médica Veterinaria, Especialista en Epidemiología de Campo.

Coordinadora, Programa Nacional de Control de Enfermedades Zoonóticas, Argentina.nacasas@msal.gov.ar

10 Médica Veterinaria, Especialista en Epidemiología de Campo, Zoonosis. Dirección de Programación Sanitaria SENASA, Argentina. pfamiotti@gmail.com

\section{Leishmaniasis visceral: senderos que confluyen, se bifurcan}

\section{Visceral leishmaniasis: paths that converge and divide}

\author{
Salomón, Oscar Daniel1; Mastrángelo, Andrea Verónica²; Santini, María \\ Soledad3; Ruvinsky, Silvina4; Orduna, Tomás5; Sinagra, Angel6; Luna, \\ Concepción ${ }^{7}$; Riarte, Adelina ${ }^{8}$; Casas, Natalia9 ; Amiotti, Paola ${ }^{10}$
}

RESUMEN La leishmaniasis visceral urbana es una zoonosis emergente en Argentina. En América es producida por Leishmania infantum, con el perro como reservorio principal e insectos flebotomíneos como vectores. En este artículo se presenta el conocimiento acumulado a partir de su emergencia y dispersión en el país, por los referentes del Programa Nacional de Leishmaniasis, en el diagnóstico clínico y de laboratorio, tratamiento, biología de vectores, manejo de reservorio, y el conflicto generado con las acciones recomendadas en relación con los perros infectados. La detección temprana y el tratamiento precoz, con estrategias descentralizadas y horizontales, contribuirán a disminuir la morbimortalidad asociada a la leishmaniasis visceral. El control de su transmisión y dispersión requiere de un manejo ambiental integral y la tenencia responsable de perros. Se discuten los intereses y discursos en conflicto generados por la leishmaniasis visceral en el marco de la relación humano-perro, proponiendo la búsqueda de un discurso consensuado de riesgo.

PALABRAS CLAVE Leishmania infantum; Lutzomyia longipalpis; Diagnóstico; Medicina Clínica; Percepción Social; Mascotas.

ABSTRACT Urban visceral leishmaniasis is an emerging zoonosis in Argentina. In the Americas the disease is produced by Leishmania infantum, with dogs as the primary reservoir and phlebotomine sandflies as the vectors. This article presents the experience acquired by professionals from the National Leishmaniasis Program in Argentina as visceral leishmaniasis has emerged and spread, especially in clinical and laboratory diagnosis, disease treatment, vector biology, reservoir management, and conflicts regarding recommendations for infected dogs. Early detection and treatment, along with decentralized and horizontal strategies, will contribute to the decrease in morbidity and mortality associated with visceral leishmaniasis. Control over the transmission and spread of the disease requires integral environmental management and responsible dog ownership. The interests and discourses put into conflict by visceral leishmaniasis are discussed in the framework of the human-dog relationship, and the search for a consensus-based risk discourse is proposed.

KEY WORDS Leishmania infantum; Lutzomyia longipalpis; Diagnosis; Clinical Medicine; Social Perception; Pets. 


\section{INTRODUCCIÓN}

El término leishmaniasis se utiliza para denominar a un conjunto de manifestaciones clínicas producidas por diferentes agentes etiológicos, parásitos tripanosomatídeos del género Leishmania (Kinetoplastida: Trypanosomatidae). El vector es un insecto flebótomo (Diptera: Psychodidae: Phlebotominae). El humano contrae la enfermedad cuando es picado por una hembra del vector, que previamente ha picado a un mamífero infectado. Las dos formas clínicas presentes en el país son la leishmaniasis tegumentaria (cutánea y mucosa) y la leishmaniasis visceral, producidas por parásitos distintos, y con diferentes vectores, reservorios, escenarios eco-epidemiológicos e impactos en la salud pública (1).

La leishmaniasis tegumentaria (LT), ya registrada en Argentina en 1916, se encuentra desde el año 2002 en un período inter-epidémico registrando entre 100 y 150 casos por año, principalmente en Salta y Chaco, pero manteniendo casos y brotes esporádicos limitados en toda la zona endémica, que incluye también a Jujuy, Catamarca, Tucumán, Formosa, Santiago del Estero, Corrientes y Misiones. La LT se transmite en zonas selváticas, rurales y suburbanas. El agente más frecuente y asociado a brotes es Leishmania braziliensis. Aunque diferentes especies de mamíferos se han encontrado infectados, ninguno ha cumplido con los criterios para ser incriminado como reservorio primario en el país (2).

La leishmaniasis visceral urbana (LV), producida en América por Leishmania infantum (syn. chagasi) desde su primer registro autóctono comprobado en el país en el año 2006, ha presentado un total de 104 casos registrados principalmente en Misiones, aunque se notificaron casos humanos también en Corrientes, Santiago del Estero y Salta, siendo además vulnerables por presencia del vector, Formosa, Chaco y el norte de Entre Ríos. Los casos han sido tratados en su totalidad, aunque en algunas áreas de transmisión debe aún mejorarse el diagnóstico oportuno, registrándose en el país una letalidad anual que varía entre el 7 y el 11\%. La dispersión por tránsito y tráfico de mascotas ha generado la presencia de perros infectados, el principal reservorio urbano, en gran parte del territorio nacional. El vector urbano es Lutzomyia longipalpis y en la región del Chaco participaría Migonemyia migonei (3-6).

Ambas leishmaniasis son de denuncia obligatoria por el médico, el veterinario y el laboratorista (Ley Nacional 15.465/1960, Decreto PE 3.640/1964, Ley de Policía Sanitaria 3.959/1900, Decreto 27.342/44 y Resolución SENASA 422/2003). A partir de la emergencia de la LT epidémica, en la década de 1980 se constituyó el grupo multidisciplinario para el estudio de la leishmaniasis en Argentina. Sus integrantes promovieron en 1999 la creación del Programa Nacional de Leishmaniasis (PNL), y asumieron la conducción y referencia del mismo. De esta manera, se constituyó un programa operativo de salud para una enfermedad emergente/re-emergente, coordinado por investigadores especialistas en el tema. Este aspecto cobra importancia teniendo en cuenta que la leishmaniasis está catalogada por la Organización Mundial de la Salud (OMS) como enfermedad sin estrategias de control conocida, desatendida, y que además presenta una incidencia estimada de 200.000400.000 casos/año en el mundo con una tasa de letalidad estimada de 10\% (7). Por ello, los integrantes del PNL realizan reuniones periódicas continentales de referentes, para revisar las evidencias experimentales, y ratificar o rectificar las normativas programáticas según las estrategias de control que hayan demostrado mayor efectividad hasta el momento. El PNL cuenta con las herramientas de diagnóstico y tratamiento y garantiza que se encuentren disponibles en forma gratuita para toda la población.

A partir del intercambio de información entre países, cuando en el año 2000 la LV urbana llegó a Mato Grosso do Sul en Brasil y a Asunción en Paraguay, en su doble condición de investigadores y agentes del programa de control, los referentes socializaron el alerta en medios académicos y asistenciales, y construyeron al mismo tiempo la percepción del sistema de salud sobre la enfermedad y las estrategias para enfrentarla. Sin embargo, sabemos que la salud-enfermedad-atención es un proceso social en el que no solo participan sus referentes científicos sino que depende de múltiples factores, así como puede ser analizada desde múltiples dimensiones $(8,9)$. 
En relación con la LV urbana en Argentina, aunque existían antecedentes aislados de casos (10), el fenómeno epidémico urbano ocurrió en localidades sin historia de transmisión. En consecuencia, como suele ocurrir con los eventos emergentes infecciosos en salud, el primer contacto (el "¿leishma qué?") y la posterior construcción de la enfermedad se hace usualmente a partir de la presentación y representación hecha por la prensa. La LV fue así armada sobre criterios de noticia antes que de información sobre salud: conflicto y drama, riesgo de muerte generalizada, culpabilización del Estado, de las corporaciones innominadas -cambio climático-, identificación-estigmatización de los enfermos, de sus barrios, su otredad. Y es a partir de la LV construida por los medios, nominada por primera vez, que luego se resignifican saberes previos confluyentes pero no específicos, como el de "enfermedades transmitidas por mosquitos" o el de leishmaniasis cutánea en las zonas endémicas (11).

En consecuencia, para la LV urbana, mientras los referentes del PNL desde el año 2000 la construyen a partir del riesgo de emergencia, transmisión y vulnerabilidad, fuera del sistema de salud, a partir del 2006 la LV se construye desde el conflicto que rodea al manejo del reservorio canino y la eutanasia, antes que desde el problema de salud pública. El discurso que se genera sobre la LV denota así una enfermedad antropozoonótica, pero usualmente connota un campo de divergencia de intereses públicos, privados corporativos y comunitarios; de competencia entre el Estado, los profesionales liberales, los laboratorios y las protectoras de animales hablando en nombre de "la gente"; de creación de antinomias artificiales individuo-sociedad, reservorio-paciente/cliente-mascota; una arena de legitimación-deslegitimació 
desarrollar enfermedad sintomática aumenta con la desnutrición, la infección VIH y en niños inmunocomprometidos (1,13-16).

Las complicaciones infecciosas (en general bacterianas) y las hemorragias son las principales causas de mortalidad (1,13-16).

\section{Características clínicas de la leishmaniasis visceral en Argentina}

Desde 2006 hasta 2011 se ha realizado un registro nacional de casos de LV en Argentina en la población pediátrica. Se registraron 30 pacientes, la mediana de la demora del inicio de los síntomas hasta el diagnóstico fue de 2 meses (rangos 1-8 meses). La mayoría tuvo entre 2 y 3 consultas previas en centros de Atención Primaria de la Salud (APS), donde no hubo sospecha diagnóstica de LV $(4,17)$.

En 45 pacientes adultos evaluados, la mediana de demora al diagnóstico fue de 2 meses (rangos 1-14 meses) (18). Estos datos muestran las oportunidades perdidas en el diagnóstico de esta patología. Según informes recientes de Brasil, el diagnóstico más allá de los 60 días es factor de riesgo de mortalidad. También fue factor de riesgo de complicaciones y mortalidad la mediana de edad en niños (12,5 meses) y en adultos (45,7 años). Un factor predictivo de mortalidad en adultos es el antecedente de etilismo y desnutrición $(19,20)$.

\section{Dificultad en el diagnóstico clínico}

Lamentablemente la LV es percibida habitualmente como un problema de baja prioridad por parte de las autoridades gubernamentales, la sociedad y, en algunas instancias, por los propios pacientes. Los factores que contribuyen a esta percepción son, entre otros: afectación de grupos de bajos recursos que habitan en áreas periféricas, la coexistencia de otros problemas de salud que focalizan la atención de los servicios sanitarios, recursos limitados en infraestructura asistencial, falta de información acerca de la magnitud real o de la naturaleza del problema, falta de conocimiento acerca de los puntos más efectivos de intervención y escaso entrenamiento del personal de salud en esta patología (1).
La falla en la detección precoz e instauración del tratamiento oportuno se relaciona con un incremento en la morbimortalidad. La OMS establece como fundamental la capacitación del personal de salud que asiste población en riesgo de la enfermedad en aspectos de tratamiento y prevención. Los centros de APS son el primer contacto que habitualmente tienen los pacientes con el sistema de salud y es allí donde debe mantenerse el alerta y la sospecha diagnóstica de LV en áreas endémicas. Se debe sostener así la inversión en la formación de recursos humanos adecuados para lograr un objetivo prioritario, que es disminuir la mortalidad, a partir de la instalación del pensamiento sobre esta enfermedad emergente en el diagnóstico diferencial de cuadros febriles con epidemiología compatible. A ello sin dudas se debe sumar la accesibilidad a los métodos diagnósticos específicos y la provisión regular de las drogas necesarias para su tratamiento $(1,21)$.

\section{DIAGNÓSTICO DE LA LEISHMANIASIS VISCERAL}

Las características clínicas de la LV son comunes a diversas enfermedades febriles como la malaria, la esquistosomiasis, la fiebre tifoidea y otras infecciones sistémicas. Los signos y síntomas son útiles, junto al nexo epidemiológico, para definir al paciente como sospechoso, pero insuficientes para establecer un diagnóstico definitivo. Por ello es imprescindible utilizar métodos de laboratorio precisos. La detección temprana, seguida de un tratamiento adecuado, es fundamental para el control.

El diagnóstico serológico debe valorarse asociado a la clínica y la epidemiología. En nuestro país, la prueba serológica recomendada por el Programa Nacional de Leishmaniasis es la inmunocromatografía con el antígeno rK39, el cual ha mostrado altos valores de sensibilidad (88-96\%) y especificidad (96-100\%) (22-24).

La serología tiene como limitante la incapacidad de diferenciar entre enfermedad activa e infección asintomática; la reactividad serológica se mantiene por años, no siendo de utilidad en las recaídas. Además los pacientes 
asintomáticos provenientes de regiones endémicas pueden tener reactividad serológica, pero no requieren tratamiento.

Los métodos parasitológicos son el "gold estándar". La visualización directa de amastigotes en frotis de tejidos, su desarrollo en medios in vitro o in vivo, o la detección de ADN por reacción en cadena de la polimerasa (PCR) permiten un diagnóstico conclusivo. El aspirado de médula ósea es el método más utilizado (punción esternal en adultos y de cresta ilíaca en niños).

El frotis es un método adecuado, simple de implementar para el diagnóstico de rutina y posee una alta especificidad, pero la sensibilidad varía según la capacidad del microscopista y del material a analizar (bazo 93-99\%, médula ósea $53-86 \%$ o ganglio linfático $53-65 \%)(25,26)$. Un resultado positivo por frotis realizado por un operador calificado confirma un caso de leishmaniasis (especificidad 100\%).

El cultivo y la inoculación en animales susceptibles, aumentan la sensibilidad del diagnóstico y permiten el aislamiento parasitario. No se utilizan como diagnóstico de rutina, ya que es necesario contar con infraestructura apropiada y personal capacitado.

La PCR es útil para el diagnóstico y para la tipificación de las cepas circulantes. La detección de ADN del parásito en aspirados de médula ósea es más sensible que el examen microscópico. Las muestras a procesar pueden ser: biopsias de lesiones, aspirados de ganglios linfáticos, punciones de médula ósea (PAMO), extendidos de frotis teñidos con Giemsa, cultivos de parásitos y órganos de animales infectados $(27,28)$.

La LV es una enfermedad oportunista importante en pacientes inmunocomprometidos, especialmente en aquellos infectados por el VIH. En individuos inmunocompetentes, la sensibilidad de las técnicas serológicas es del $87-93 \%$, mientras que en pacientes con LV y coinfección por VIH la seronegatividad es mayor al 40\%, por ello, el diagnóstico parasitológico es de relevancia (29). Un resultado serológico negativo no descarta un diagnóstico de LV en pacientes infectados por VIH. Sin embargo, un resultado positivo a títulos bajos tiene valor diagnóstico sumado a la clínica. La visualización de parásitos o detección de ADN en muestras tisulares constituye el método de elección, aunque la PCR es más sensible y permite el seguimiento posterapéutico (30-32).

La Figura 1 muestra el algoritmo diagnóstico para LV en el que se recomienda realizar estudios serológicos y parasitológicos. Si bien en situación epidemiológica de brote se puede instaurar el tratamiento con clínica compatible y serología reactiva para LV, es aconsejable remitir muestras tisulares para la visualización de amastigotes o la detección de ADN parasitario.

Horizontalizar el diagnóstico permite la integración y permanente colaboración de los laboratorios efectores con los referentes provinciales y nacionales. La capacitación en la lectura de frotis permite minimizar los resultados falsos negativos y positivos, y reduce el tiempo del diagnóstico posibilitando una respuesta terapéutica temprana. Los laboratorios de la red deben integrarse a un programa de control tanto de procedimientos como de resultados.

\section{BIOLOGÍA DE LOS VECTORES}

Los insectos que vectorizan las leishmanias son dípteros de la familia Pshycodidae (subfamilia Phlebotominae) Ilamados comúnmente flebótomos. Son de tamaño pequeño: miden entre 2 y $4 \mathrm{~mm}$. Son organismos holometábolos; su ciclo de vida, que se extiende por unas 11 semanas, se desarrolla en 4 estadios: huevo, larva, pupa y adulto. Los tres primeros estadios se desarrollan en suelos húmedos, ricos en materia orgánica de la que se alimentan, en tanto que, en el estado adulto, aéreo, se alimentan de líquidos vegetales. Las hembras solo ingieren sangre para el desarrollo de los huevos. En general los adultos son de actividad vespertina, aunque también presentan un pequeño pico de actividad durante el amanecer.

El principal vector del parásito que produce la LV en América es Lutzomyia longipalpis $(33,34)$. La primera cita sobre esta especie en la Argentina es de un ejemplar hembra hallado en Candelaria (Misiones) en 1951 (35). Recién en el año 2000 se vuelve a citar en nuestro país, con la captura de cuatro ejemplares machos en Corpus (Misiones) (36). Junto con este hallazgo y la aparición de casos de LV en los países limítrofes 
Figura 1. Algoritmo de diagnóstico ante casos sospechosos de leishmaniasis visceral humana.

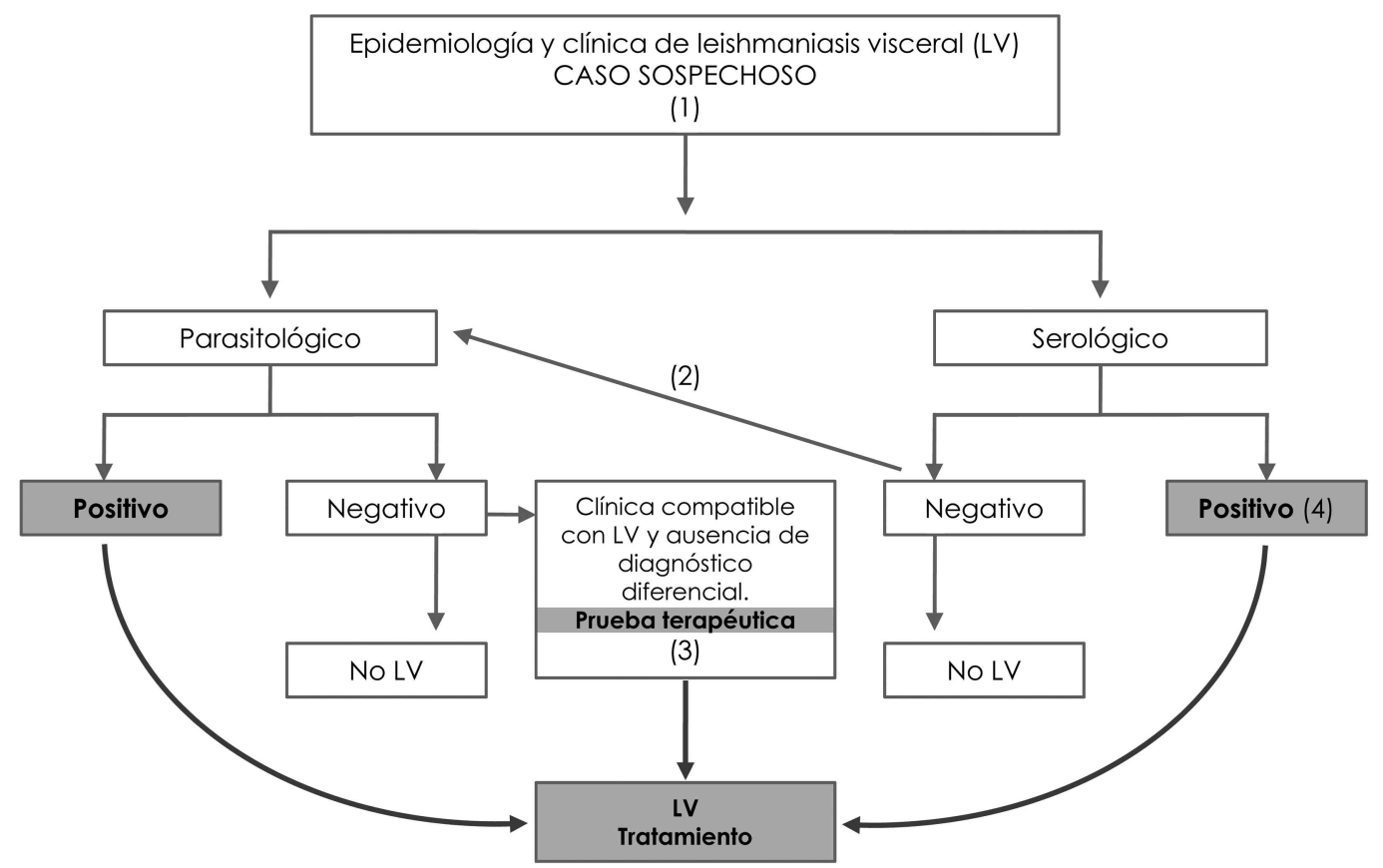

1. De ser factible todo paciente con sospecha clínica y nexo epidemiológico debe ser estudiado por métodos serológicos y parasitológicos.

2. En pacientes inmunocomprometidos, la serología tiene una baja sensibilidad (pacientes trasplantados o infectados con VIH). En estos pacientes el diagnóstico parasitológico es el indicado.

3. La prueba terapéutica puede realizarse en aquellos pacientes con clínica compatible con leishmaniasis visceral y en ausencia de diagnósticos diferenciales.

4. En situación de brote, en casos graves con epidemiología, cuadro clínico sospechoso y rk39 reactiva, se puede administrar tratamiento específico.

Fuente: Elaboración propia.

(Paraguay y Brasil), se declaró la alerta amarilla con el objeto de generar un sistema de vigilancia entomológica en área de frontera, a partir del cual se encuentra en 2004 Lutzomyia longipalpis en Clorinda (Formosa) (3), declarándose la alerta naranja para el territorio nacional. En el año 2006 ocurre en la ciudad de Posadas (Misiones) el primer caso autóctono de LV humano, en el que coexisten el parásito, el reservorio y el vector y se confirma la transmisión de LV en nuestro país (alerta roja) (4). Estudios posteriores demostraron la rápida dispersión hacia el sur del complejo parásito L. infantum - vector Lu. longipalpis reservorio Canis familiaris infectado, por el noreste del país $(37,38)$. La distribución actual comprende a Formosa, Misiones, Corrientes, Chaco y en Entre Ríos hasta la ciudad de Chajarí (6). Sin embargo, la ciudad más al sur donde se encontraron ejemplares del vector de LV fue Salto, en la República Oriental del Uruguay (39), frente a la ciudad argentina de Concordia (Entre Ríos) donde aún no se han observado ejemplares del vector (6), lo que nos podría estar advirtiendo que la distribución de Lu. longipalpis observada en la Argentina no sea aún la definitiva.

En todas las ciudades estudiadas, Lu. longipalpis mostró ser una especie adaptada al ambiente urbano. Se observó que se distribuye de forma heterogénea, con áreas de alta abundancia entremezcladas con áreas de abundancia baja o presencia nula (40). Estas áreas, dinámicas en el espacio y en el tiempo, se ven definidas 
principalmente por las características microambientales (41).

En la región chaqueña de nuestro país, a partir de la ausencia de Lu. longipalpis, la presencia de reservorios infectados y la ocurrencia esporádica de casos humanos, se propuso como posible vector de $L$. infantum a otra especie de flebótomo: Mygonemyia migonei (5).

Para controlar y prevenir la continua dispersión de los vectores es necesario tener en cuenta que las intervenciones con el solo uso de insecticidas mostraron poca efectividad, permitiendo realizar bloqueos focales de cobertura reducida y de corta duración (42). En este sentido, considerando la rápida y amplia dispersión de los vectores, el aumento de casos humanos, el aumento de la dispersión y de los casos caninos, se refuerza la idea de trabajar la leishmaniasis como un todo, realizándose acciones de prevención y control de manera integrada sobre el ambiente, entendiendo al mismo ambiente como el conjunto de valores naturales, culturales y sociales con el fin de trabajar sobre la salud previniendo la enfermedad.

\section{TENENCIA RESPONSABLE Y MANEJO DE RESERVORIOS}

Como se ha dicho, el principal reservorio de la LV es el perro doméstico $(43,44)$. Una de las estrategias dentro del control integrado de la enfermedad es controlar los reservorios para interrumpir la circulación y dispersión geográfica de los parásitos.

La prevención está relacionada con la tenencia responsable de los caninos; para la Organización Mundial de Sanidad Animal (OIE) la propiedad responsable de un perro

\footnotetext{
...es la situación en que una persona acepta y se compromete a cumplir una serie de obligaciones, encaminadas a satisfacer las necesidades comportamentales, ambientales y físicas de un perro y a prevenir los riesgos (agresión, transmisión de enfermedades o heridas) que el animal pueda presentar para la comunidad, para otros animales o para el medio. (45)
}

Es necesario el fortalecimiento de la gestión local para la promoción de la tenencia responsable; con estas acciones trabajamos en la prevención mediante el cambio de hábitos en la población. La tenencia responsable tiene implicancias en diferentes ámbitos de la vida cotidiana, lo que conlleva a trabajar sobre aspectos ligados con los modos de vida de las personas y con las condiciones en las que estas se desarrollan. El médico veterinario debe actuar como concientizador y, a su vez, la tenencia responsable debería estar incluida dentro de los contenidos curriculares en colegios de nivel primario y secundario.

Se debe crear una legislación nacional que contemple las normas establecidas por los organismos internacionales (OIE-OPS/OMS), basada en un programa integral, que abarque la educación ciudadana sobre tenencia responsable de animales, y que incluya: identificación y registro de animales y tenedores, uso de libreta sanitaria oficial, normativa sobre establecimientos de venta o cuidado de animales de compañía, criaderos, paseadores, establecimientos veterinarios, exposiciones, refugios o albergues caninos, control de animales vagabundos, preservación del medio ambiente, riesgo sanitario, creación y fortalecimiento de los centros de zoonosis ya existentes, y sanciones a los infractores (45). En el año 2011, el Poder Ejecutivo Nacional creó por decreto 1.088/11 el Programa Nacional de Tenencia Responsable y Sanidad de Perros y Gatos (46); parte de sus objetivos estarían implicados en las acciones antes mencionadas.

Dentro de las medidas de control de la población canina, la esterilización quirúrgica es una de las actividades de mayor impacto en los programas de salud pública y tal vez sea una de las acciones más conocidas por la sociedad en general (47). Hay que tener en cuenta que la cobertura de esterilización quirúrgica debe ser alta para mantener estable la población canina (48), lo que resulta complicado para algunos sistemas locales. Por este motivo, como se mencionó con anterioridad, un adecuado control debe estar siempre acompañado por cambios en las conductas y comportamientos de la población humana.

Con relación al tratamiento farmacológico en perros con LV, lo más frecuente es que solo se observe una mejoría clínica pero siguen 
siendo fuente de parásitos para los vectores. El tratamiento con drogas utilizadas en humanos aumenta el riesgo de generación de cepas resistentes a dichos medicamentos (1). En el continente europeo, donde se ha utilizado intensamente el tratamiento en perros, se observa un aumento en la prevalencia de LV humana y canina en los últimos años $(49,50)$. Otras medidas como los collares o pipetas repelentes son útiles para proteger animales sanos $(51,52)$ pero no garantizan la interrupción de la transmisión por parte de los perros infectados.

La eutanasia del perro infectado se recomienda con el fin de eliminar la oferta parasitaria a los vectores y su eficacia es mayor cuando se aplica en los primeros casos, evitando la instalación de la enfermedad en una zona. Si bien en algunos focos se han observado disminuciones en la incidencia de casos humanos de LV después de sacrificios masivos de caninos $(53,54)$, en otras áreas esta estrategia fue una medida insuficiente para erradicar la LV canina $(55,56)$. La principales razones de falla como método de control son:

1. poca aceptación por parte de la población en general;

2. baja cobertura debido a la baja sensibilidad de las pruebas diagnósticas;

3. retraso en la detección y eutanasia de los perros;

4. los animales sacrificados son frecuentemente reemplazados por perros no infectados susceptibles de adquirir la enfermedad al poco tiempo (57).

El manejo del reservorio de la LV urbana es complejo y debe desarrollarse de manera integrada y en forma intersectorial. Es imprescindible que toda medida de control que se realice deba ir acompañada de políticas públicas que le den sustento legislativo a las acciones por emprender.

\section{AMORES PERROS}

Familia y naturaleza son espacios socialmente diferenciados. El reservorio de la LV, el perro, es el animal doméstico más frecuente en Argentina (58). La domesticación fue un proceso histórico, gradual, de adaptación evolutiva donde el humano ejerció selección de determinadas características en poblaciones animales o vegetales. El perro está entre las primeras especies domesticadas hace unos 15.000 años. Por ser un animal doméstico del que no se obtiene lucro, se lo agrupa dentro de los animales de compañía (59). La tenencia doméstica de animales entrecruza el orden natural con el humano/cultural (60). En los párrafos que siguen, este enfoque será la clave para la comprensión de la emergencia de la LV en Posadas y su vigilancia en la región del nordeste argentino (NEA).

\section{Ambiente y ambientalización en la emergencia de la leishmaniasis visceral en Posadas}

Desde la década de 1980 al presente, la ambientalización (61) en Misiones avanzó paralelamente en dos frentes: el movimiento urbano antirepresas en Posadas $(62,63)$ y las políticas de conservación de la selva paranaense y su fauna en el resto de la provincia (64). Sostenemos que, como parte de la ambientalización, al momento de la emergencia de LV en Posadas en 2006 (4), la definición del estatus doméstico del perro y su rol ambiental estaban siendo redefinidos. Ferrero (64) ha descrito la progresiva disminución de la caza con perros por resultar impropia al cuidado ambiental. En consonancia con la ambientalización de la ciudadanía de los posadeños esto implicó un cambio en el tipo social de perro demandado: se buscan cachorros de razas pequeñas para compañía (65) o perros robustos para resguardo del patrimonio (66).

El perro de compañía o perro guardián vive dentro o cuida la casa, adoptando el lugar del femenino hegemónico dentro de lo doméstico y aburguesan el consumo del hogar que habitan. En este contexto, la cría informal y el tráfico transfrontera satisfacen la demanda de un mercado creciente. Esta asociación entre ciertas razas de perro y el gasto de los hogares permite pensar que no todos los riesgos para la salud provienen de la pobreza (67). 


\section{Tipos sociales de perro}

Encontramos en Posadas 4 tipos socioculturales de perros.

Tipo 1. Los más desprotegidos de la sociedad: Una asociación civil, El Refugio, alberga 200 perros en un lote en comodato sin infraestructura en la periferia de Posadas y ha dado en adopción 5.273 perros entre 2001-2011 (68); 16 animales fueron diagnosticados con LV y la asociación es activa militante contra la eutanasia.

Tipo 2. Criaderos formales e informales de perros de compañía y guardianes $(65,66)$ : Los perros tenidos para reproducción y venta pasan de ser animales domésticos de compañía a ser animales de renta, por lo que debería regularlos una normativa sanitaria específica (59); en el mismo estatus regulatorio se ubicarían las residencias para cuidado de perros.

Tipo 3. Perros de búsqueda: Por ser una provincia con frontera internacional, las fuerzas de seguridad intervienen en el control de la circulación con perros detectores de narcóticos o divisas.

Tipo 4. Un cachorro de regalo: Las redes de donación son un espacio informal de relaciones sociales no permanentes que podrían estar distribuyendo animales parasitados (66). Los animales de compañía tienen mayor movilidad geográfica. Un cachorro regalado como animal de compañía a un estudiante de Posadas que se radicó en Santo Tomé estuvo involucrado en la emergencia de LV en Corrientes. Es habitual que los posadeños lleven a sus mascotas a las playas de Ituzaingó (Corrientes).

Cada tipo ameritaría una regulación sanitaria específica, siendo relevante incidir en el control reproductivo de los animales parasitados, la certificación negativa de los cachorros y la circulación (nacional y transfronteriza).

\section{Dilemas morales de la vigilancia epidemiológica}

Los tipos sociales de perros domésticos implican relaciones sociales e intereses económicos particulares que eclosionan de varias maneras al implementar la vigilancia epidemiológica.

La vigilancia sanitaria incluye la voluntad pero debe optar un criterio normativo. Todas las ramas del Derecho tienen interés sobre los animales de compañía: la ambiental, la penal, la internacional, la mercantil y la civil (59). Los criaderos y residencias, por ser una actividad de renta, podrían quedar sujetos a la normativa federal, pero la normativa sanitaria de animales de compañía es, en Iberoamérica, de competencia municipal (59).

La Declaración Internacional de los Derechos del Animal (1978), de claro paralelismo con la Declaración de los Derechos del Hombre (1945) es un punto de inflexión para el control de las antropozoonosis. Centradas en ella, las normas municipales analizadas apuntan, para proteger las obligaciones de los propietarios humanos con los animales de compañía, a su identificación, censo y registro. La identificación de perro y propietario es una regulación necesaria para prohibir el abandono y exigir el cumplimiento de los derechos del animal $(59,69)$. Los derechos de la naturaleza son sin duda una herida narcisista al antropocentrismo (70), pero ¿alcanzan a los parásitos que enferman al hombre? Puestos frente a frente un hombre y un perro abandonado con leishmaniasis, ¿tienen los mismos derechos si tratar al perro puede generar parásitos resistentes al único tratamiento disponible para humanos? Más aún, si el perro con leishmaniasis tiene dueño, ese dueño y el veterinario que lo atiende ¿pueden definir autónomamente entre tratamiento y eutanasia?

\section{CONCLUSIÓN}

En las secciones precedentes hemos revisado someramente el corpus de conocimiento sobre LV urbana acumulado, por los referentes académicos-programáticos, desde el primer alerta de emergencia en Argentina y su transferencia a los equipos de salud. Pero también se infiere del texto que los referentes de LV, como los de cualquier patógeno emergente, no deben solo nombrar, estandarizar y coordinar la vigilancia, garantizar la accesibilidad a un diagnóstico temprano, tratamiento oportuno, y generar las medidas de prevención adecuadas con acciones basadas en evidencias científicamente validadas. La dinámica comunicacional de las enfermedades 
emergentes y epidémicas implica que, al abandonar las secciones de salud de los medios para crecer desde las secciones de información general y luego multiplicarse en citas resignificadas en múltiples contextos en Internet, la percepción se construye desde el conflicto, en el caso de la LV concentrado en el tema canino. Luego, si se asume la responsabilidad como sanitaristas, las voces legitimadas del sistema deben también hacer el ejercicio permanente para recontextualizar la discusión, atentos a los cambios de la epidemia en tiempo y espacio, buscando el equilibrio, evitando posiciones extremas, haciendo el ejercicio voluntario de escuchar a los diferentes agentes, y buscando caminos de compromiso y consenso entre lo necesario y lo factible.

Desde el ámbito de las ciencias, la aparición de una situación novedosa genera expertos al asalto de nuevas fuentes de financiamiento, de nuevos modelos de trabajo, de individuos reificados como casos o muestras. Aun contra los propios intereses inmediatos de los referentes, desde la perspectiva sanitaria, se debe entonces trabajar para asignar al problema la prioridad que merece en cada escenario epidemiológico, sin magnificarlo, y reorientando los esfuerzos hacia las necesidades reales del sistema y la salud pública.

En el ámbito político-sanitario los eventos agudos y colectivos de enfermedades biológicas no provocan pero exponen las enfermedades sociales y ausencias del sistema de salud, las inequidades, los signos y síntomas crónicos de las patologías estructurales que lo afectan. Por ello, además del trabajo específico de investigación de servicios para mejorar el sistema, se debe invertir tiempo en retornar a un discurso responsable frente a la negación del "no pasa nada", a la naturalización del "siempre estuvo" y "llegó para quedarse", del exitismo de corta duración por intervenciones parciales de fenómenos que sabemos multifactoriales. En lo operativo se debe también reiterar la necesidad de evaluación de las "campañas" comunicacionales y de intervención, destacar que la asignación de responsabilidades no implica distribución homogénea de responsabilidades (del individuo al Estado), y recordar que en la sociedad capitalista los medios de difusión tienen sus objetivos "noticia" diferentes de la educación para la salud (11).
Desde los sectores con intereses económicos explícitos o implícitos -para la LV los relacionados fundamentalmente con canes (laboratorios, puestos de venta de productos, criadores, corporaciones profesionales, y sus gestores políticos o mediáticos) - es usual que se pase de la negación del alerta (disease awarness) cuando no se tiene respuesta, a su sobredimensionamiento con fines comerciales (disease mongering) afirmando que el problema es de vieja data, que está "todo el tiempo en todos lados". En este caso se debe recontextualizar la discusión apelando a las evidencias experimentales que han demostrado mayor efectividad hasta el momento, y requiriendo una importante dosis de competencia social de los referentes del sistema para identificar qué, dónde y en qué términos se discute. Los principales ejes de dicha discusión se pueden resumir en:

a) Diferenciar objetivos de salud individual y salud colectiva: perros tratados que mejoran la clínica y continúan siendo fuente de parásitos por más tiempo, tratamientos caninos que pueden inducir resistencia del parásito a las drogas utilizadas para tratar la LV humana, repelente como protección individual cuando el impacto en la transmisión es función del grado de cobertura con repelentes de la población de reservorios expuesta (herd effect).

b) Señalar aquellas soluciones ofertadas desde lo comercial que requieren todavía experimentación o que implican costos directos e indirectos inaccesibles para un programa de salud: vacunas (en fase II, con aplicaciones sucesivas o necesidad de pruebas específicas para diferenciar vacunado de infectado), repelentes masivos (costo de implementación en terreno, royalties, packaging y publicidad), y fumigaciones masivas que además de su costo financiero no demostraron ser efectivas para el control del vector (42).

c) Exigir rigor metodológico de pruebas experimentales frente al empirismo, al manejo publicitario de datos (gráficas de impacto visual), o al discurso parcializado: el punto final experimental debe ser la interrupción de la transmisión o la mitigación de la morbimortalidad humana; la promoción de productos antivectoriales tiene sentido donde hay vectores y no en las grandes ciudades con población con 
poder adquisitivo pero sin vector; explicitar que las estrategias de control vectorial efectivas se basaron en el uso del DDT u otros productos prohibidos, y en relación con los tratamientos caninos informar el porcentual de recidivas y la persistencia parasitaria.

d) Estar vigilantes ante el discurso que usa como determinantes las condiciones socioeconómicas o de origen: se identifica al ciudadano de otro país/provincia como caso importado y se lo enmarca en los medios mostrando la amenaza, pero se obvia la introducción de perros de raza importados para su reproducción que dispersan el parásito; se estigmatiza a humanos por inmunosuprimidos, alcohólicos, ancianos, pobres-desnutridos (personas negligentes en lugar de personas negligenciadas con enfermedades negligenciadas), se extraña y culpa a los humanos para minimizar el riesgo asociado a los perros infectados, un otro más cercano del que somos responsables.

Este permanente ejercicio de recontextualización frente a los distintos actores con intereses específicos refiere a la voz última, pero no última voz, que debe ser escuchada: los profesionales que entienden el concepto de salud humana-animal como objetivo de supervivencia global, las ONG proteccionistas con capacidad de discusión racional, y fundamentalmente la voz heterogénea de la comunidad. Estos agentes reciben todos los discursos precedentes y los deben yuxtaponer y reelaborar a partir de sus propios saberes y del afecto legítimo que tienen por sus mascotas. Por eso, desde el Programa Nacional de Leishmaniasis confluimos con el Programa Nacional de Tenencia Responsable, y en este trabajo y en los grupos de investigación integramos en forma constitutiva la visión de las ciencias sociales a la biomédica y bioecológica. Sin embargo, en el medio, como ya se señaló, están los medios de difusión masiva, amplificando las controversias, equiparando fuentes de información diferentes, ofreciendo prestigio instantáneo a "expertos" de Internet, deslegitimando a los referentes oficiales per se por ser la voz del Estado, concentrados en la cuantificación de casos, en su identificación individual, fomentando el individualismo en la sociedad de riesgo global (71), alentando la medicalización de lo cotidiano (la comida, el ocio, el afecto, la mascota como un objetivo de salud) pero desacreditando a su vez a la medicina tradicional al promover estrategias alternativas sin validación (healthism) (11).

Por ello, con el objeto de construir un programa de salud, y no solo de enfermedad, se deben encontrar los puntos de acuerdos básicos y necesarios, atendiendo a las voces de buena voluntad. En esta búsqueda de contextualizar el discurso y las acciones, asumiendo las responsabilidades del Estado como garante de la salud de sus ciudadanos, se necesita transformar la percepción de saberes que se bifurcan en saberes que confluyen, y llegar a la comunidad con un discurso común de riesgo. Estos puntos mínimos, para la LV, comprenden:

a) que el Estado garantice el diagnóstico temprano y tratamiento oportuno de la LV humana, horizontalizando y supervisando las acciones;

b) reconocer la LV como enfermedad humana con letalidad potencial, no solo como problema canino;

c) comunicar el riesgo asociado al animal infectado con o sin síntomas, sin minimizarlo, entendiendo que este conocimiento es también un derecho humano, e incluyendo en ese riesgo el traslado de mascotas por intermedio de las redes sociales y comerciales; $y$

d) generar acciones efectivas, secuenciales, multisectoriales, adaptadas a cada escenario epidemiológico, donde el combate efectivo al vector implica un ambiente público y privado saludable, y no "campañas" con insecticidas caros desde lo financiero, ambiental y sanitario, y de muy corta duración y efecto.

\section{AUTORÍA}

La Introducción y Conclusión han sido escritas por Oscar Daniel Salomón, la sección “Manejo de pacientes y problemas relacionados al diagnóstico clínico y socialización de pacientes con la leishmaniasis visceral" por Silvina Ruvinsky y Tomás Orduna, el "Diagnóstico de la leishmaniasis visceral" por 
Concepción Luna, Adelina Riarte y Angel Sinagra, "Biología de los vectores" por María Soledad Santini, "Tenencia responsable y manejo de reservorios" por Natalia Casas y Paola Amiotti, y "Amores perros" por Andrea Verónica Mastrángelo. Las opiniones vertidas en cada sección son responsabilidad del o los autores correspondientes.

\section{REFERENCIAS BIBLIOGRÁFICAS}

1. World Health Organization. Control of the Leishmaniases, WHO Technical Report Series $N^{\circ}$ 949. Geneva: WHO; 2010.

2. Salomón OD, Quintana MG, Rosa JR. Ecoepidemiología de la leishmaniasis cutánea en Argentina. Salud(i)Ciencia. 2008;16(5):514-520.

3. Salomón OD, Orellano PW. Lutzomyia longipalpis in Clorinda, Formosa province, an area of potential visceral leishmaniasis transmission in Argentina. Memorias do Instituto Oswaldo Cruz. 2005;100(5):475-476.

4. Salomon OD, Sinagra A, Nevot MC, Barberian G, Paulin P, Estevez JO, Riarte A, Estevez J. First visceral leishmaniasis focus in Argentina. Memorias do Instituto Oswaldo Cruz. 2008;103(1):109-111.

5. Salomón OD, Quintana MG, Bezzi G, Morán ML, Betbeder E, Valdéz DV. Lutzomyia migonei as putative vector of visceral leishmaniasis in La Banda, Argentina. Acta Tropica. 2010;113(1):84-87.

6. Gould IT, Perner MS, Santini MS, Saavedra SB, Bezzi G, Maglianese MI, Antman JG, Gutiérrez JA, Salomón OD. Leishmaniasis visceral en Argentina: análisis de la notificación y situación vectorial en las provincias de Entre Ríos, Santa Fe y Santiago del Estero, 2011. Medicina (Buenos Aires). En prensa 2012.

7. Alvar J, Vélez ID, Bern C, Herrero M, Desjeux P, Cano J, Jannin J, den Boer $M$, the WHO Leishmaniasis Control Team. Leishmaniasis worldwide and global estimates of its incidence. PLoS One [Internet]. 2012 [citado 10 jun 2012];7(5):e35671. Disponible en: http://www.plosone.org/article/info \%3Adoi\%2F10.1371\%2Fjour nal.pone.0035671.

8. Menéndez E. Aproximación crítica al desarrollo de la antropología médica en América Latina. Nueva Antropología. 1985;7(28):12-27.

9. Mastrángelo AV, Salomón OD. Contribución de la antropología a la comprensión ecoepidemiológica de la Leishmaniasis Tegumentaria
Americana en las "2000 hectáreas", Puerto Iguazú, Misiones, Argentina. Revista Argentina de Salud Pública. 2010;1(4):6-13.

10. Salomón OD, Rossi G, Sosa Estani S, Spinelli G. Presencia de Lutzomyia longipalpis y situación de la leishmaniosis visceral en Argentina. Medicina (Buenos Aires). 2001;61(2):174-178.

11. Petracci M, Waisbord S. Comunicación y salud en la Argentina. Buenos Aires: La Crujía Ediciones; 2011.

12. Dias-Bevilacqua $P$, Paixão $\mathrm{HH}$, Castro MCPS, Modena CM. Leishmaniose visceral: história jornalística de uma epidemia em Belo Horizonte, Brasil. Interface. 2000;4(7):83-102.

13. World Health Organization. Report of the scientific working group meeting on Leishmaniasis, TDR/SWG/04. Geneva: WHO. 2004.

14. Ministerio de Salud de la Nación. Manual de procedimientos para el control de la Leishmaniasis en Argentina. Buenos Aires: MSAL; 2006.

15. Ministerio da Saúde. Manual de Vigilância e Controle da Leishmaniose visceral. Brasilia: MS; 2003.

16. Ministerio de Salud de la Nación. Manual de Leishmaniasis Visceral Argentina 2010: Guía para profesionales de la salud. Buenos Aires: MSAL; 2010.

17. Ruvinsky S, Salomon O, Arce L , Jozami D, Altcheh J, Cappellini R, Lopez Papucci S, Riarte A, Gomez S, Borchichi L, Bologna R1, Paulin P, Pividori R, Moscatelli G, Freilij H, Chiabrando R, Gentile A, Aletti A, Gajo Gane A, Orduna T. Clinical analysis and eco-epidemiology data of pediatric visceral leishmaniasis (VL) in Argentina. International Journal of Infectious Diseases. 2010;14(Supl 1):e333-e334. Disponible en: http://www.sciencedirect.com/science/article/pii/ S1201971210022745.

18. Calvano M, Compañy L, Heredia C, Koch M, Maidana Proch A, Méndez G, Niveyro C, Silva G. Leishmaniasis visceral: características clínicoepidemiológicas de pacientes internados en un hospital de agudos. XI Congreso SADI: Libro de 
Resumenes [Internet]. Mar del Plata: SADI; 2011 [citado 10 jun 2012]. p. 13. Disponible en: http://www.sadi.org.ar/files/file/Libro\%20de $\% 20$ Resumenes\%20SADI\%202011.pdf.

19. Alvarenga DG, Escalda PM, Costa AS, Monreal MT. Leishmaniose visceral: estudo retrospectivo de fatores associados à letalidade. Revista da Sociedade Brasileira da Medicina Tropical. 2010;43(2):194-197.

20. Oliveira JM, Fernandes AC, Dorval ME, Alves TP, Fernandes TD, Oshiro ET, Oliveira AL. Mortalidade por leishmaniose visceral: aspectos clínicos e laboratoriais. Revista da Sociedade Brasileira da Medicina Tropical. 2010;43(2):188-193.

21. Romero GA, Boelaert M. Control of Visceral Leishmaniasis in Latin America-A Systematic Review. PLoS Neglected Tropical Diseases [Internet]. 2010 [citado 12 jun 2012];4(1):e584. Disponible en: http://dx.plos.org/10.1371/journal.pntd.0000584.

22. Chappuis F, Rijal S, Soto A, Menten J, Boelaert M. Meta-analysis of the diagnostic performance of the direct agglutination test and rK39 dipstick for visceral leishmaniasis. British Medical Journal [Internet]. 2006 [citado 12 jun 2012];333(7571): 723. Disponible en: http://www.bmj.com/cgi/pmidlookup?view $=$ long\&pmid $=16882683$

23. Carvalho SF, Lemos EM, Corey R, Dietze R. Performance of recombinant K39 antigen in the diagnosis of Brazilian visceral leishmaniasis. American Journal of Tropical Medicine \& Hygiene. 2003;68(3):321-324.

24. Assis TSM, Braga ASC, Pedras MJ, Barral AMP, Siqueira IC, Costa CHN, Costa DL, Holanda TA, Soares VYR, Biá M, Caldas BAJM, Romero GAS, Rabello A. Validação do teste imunocromatográfico rápido IT-LEISH ${ }^{\circledR}$ para o diagnóstico da leishmaniose visceral humana. Epidemiologia e Serviços de Saúde. 2008; 17(2):107-116.

25. Zijlstra EE, Ali MS, el-Hassan AM, el-Toum IA, Satti M, Ghalib HW, Kager PA. Kala-azar: a comparative study of parasitological methods and the direct agglutination test in diagnosis. Transactions of the Royal Society of Tropical Medicine \& Hygiene. 1992;86(5):505-507.

26. Ho EA, Soong TH, Li Y. Comparative merits of sternum, spleen and liver punctures in the study of human leishmaniasis. Transactions of the Royal Society of Tropical Medicine \& Hygiene. 1948;41(5):629-636.
27. Rodgers MR, Popper SJ, Wirth DF. Amplification of kinetoplast DNA as a tool in the detection and diagnosis of Leishmania. Experimental Parasitology. 1990;71(3):267-275.

28. Luyo-Acero GE, Uezato $\mathrm{H}$, Oshiro M, Takei K, Kariya K, Katakura K, Gomez-Landires E, Hashiguchi Y, Nonaka S. Sequence variation of the cytochrome $b$ gene of various human infecting members of the genus Leishmania and their phylogeny. Parasitology. 2004;128(5):483-491.

29. Alvar J, Cañavate $C$, Gutiérrez-Solar B, Jiménez M, Laguna F, López-Vélez R, Molina R, Moreno J. Leishmania and human immunodeficiency virus coinfection: the first 10 years. Clinical Microbiology Reviews. 1997;10(2):298-319.

30. Cota GF, Sousa MR, Demarqui FN, Rabello A. The diagnostic accuracy of serologic and molecular methods for detecting visceral leishmaniasis in HIV infected patients: Meta-analysis. PLoS Neglected Tropical Diseases [Internet]. 2012 [citado 12 jun 2012];6(5):e1665. Disponible en: http://www.plosntds.org/article/info \% 3Adoi \% 2F10.1371\%2Fjournal.pntd.0001665

31. Piarroux R, Gambarelli F, Dumon H, Fontes M, Dunan S, Mary C, Toga B, Quilici M. Comparison of PCR with direct examination of bone marrow aspiration, myeloculture, and serology for diagnosis of visceral leishmaniasis in inmunocompromised patients. Journal of Clinical Microbiology. 1994;32(3):746-749.

32. Antinori S, Calattini S, Longhi E, Bestetti $G$, Piolini R, Magni C, Orlando G, Gramiccia M, Acquaviva V, Foschi A, Corvasce S, Colomba C, Titone L, Parravicini C, Cascio A, Corbellino $M$. Clinical use of polymerase chain reaction performed on peripheral blood and bone marrow samples for the diagnosis and monitoring of visceral leishmaniasis in HIV infected and HIV-uninfected patients: a single-center, 8year experience in Italy and review of the literature. Clinical Infectious Diseases. 2007;44(12):1602-1610.

33. Le Pont F, Desjeux P. Leishmaniasis in Bolivia. Lutzomyia longipalpis (Lutz \& Neiva, 1912) as the vector of visceral leishmaniasis in Los Yungas. Transactions Royal Society Tropical Medicine \& Hygiene. 1985;79(2):227-231.

34. Lainson R, Rangel EF. Lutzomyia longipalpis e a ecoepidemiologia da leishmaniose visceral americana (LVA) no Brasil. En: Rangel EF, Lainson R. Flebotomíneos do Brasil, Rio de Janeiro: Fiocruz; 2003. 
35. Duret JP. Notas sobre flebótomos argentinos. Revista Sanidad Militar Argentina. 1952;51:534536.

36. Salomón OD, Sosa Estani S, Rossi G, Spinelli G. Presencia de Lutzomyia longipalpis y situación de la leishmaniasis visceral en Argentina. Medicina (Buenos Aires). 2001;61(2):174-178.

37. Salomón OD, Ramos LK, Quintana MG, Acardi SA, Santini MS, Schneider A. Distribución de vectores de leishmaniasis visceral en la provincia de Corrientes. Medicina (Buenos Aires). 2009;69(6):625-630.

38. Salomón OD, Fernández $M$, Santini $M$, Saavedra S, Montiel N, Ramos M, Rosa J, Szelag E, Martínez M. Distribución de Lutzomyia longipalpis en la Mesopotamia Argentina, 2010. Medicina (Buenos Aires). 2011;71(1):22-26.

39. Salomón OD, Basmajdian Y, Fernández MS, Santini MS. Lutzomyia longipalpis in Uruguay: the first report and the potential of visceral leishmaniasis transmission. Memorias do Instituto Oswaldo Cruz. 2011;106(3):381-382.

40. Fernández MS, Salomón OD, Cavia R, Pérez AA, Acardi SA, Guccione JD. Lutzomyia longipalpis spatial distribution and association with environmental variables in an urban focus of visceral leishmaniasis, Misiones, Argentina. Acta Tropica. 2010;114(2):81-87.

41. Santini MS, Fernández MS, Pérez AA, Sandoval AE, Salomón OD. Lutzomyia longipalpis abundance in the city of Posadas, northeastern Argentina: variations at different spatial scales. Memorias do Instituto Oswaldo Cruz. En prensa 2012.

42. Santini MS, Salomón OD, Acardi SA, Sandoval EA, Tartaglino LC. Lutzomyia longipalpis behavior at an urban visceral leishmaniasis focus in Argentina. Revista Instituto Medicina Tropical São Paulo. 2010;52(4):187-191.

43. Camargo-Neves VLF, Gomes A de C, Ferreira Antunes JL. Correlação da presença de espécies de flebotomíneos (Diptera: Psychodidae) com registros de casos da leishmaniose tegumentar americana no Estado de São Paulo, Brasil. Cadernos de Saúde Pública. 2001;17(5):1263-1267.

44. Moreno EC, Melo MN, Genaro O, Lambertucci JR, Serufo JC, Andrade AS, Antunes CM, Carneiro M. Risk factors for Leishmania chagasi infection in an urban area of Minas Gerais State. Revista da Sociedade Brasileira de Medicina Tropical. 2005;38(6):456-463.
45. Organización Mundial de Sanidad Animal. Control de las poblaciones de perros vagabundos. En: Código Sanitario para los Animales Terrestres. Paris: OIE; 2011. p. 409-425.

46. Argentina. Decreto 1088/2011 "Programa Nacional de Tenencia Responsable y Sanidad de Perros y Gatos". 20 jul 2011.

47. World Society for the Protection of Animals (WSPA). Guía para el manejo humanitario de poblaciones caninas: Coalición Internacional para el Manejo de Animales de Compañía [Internet]. 2007 [citado 12 jun 2012]. Disponible en: http://www.icam-coalition.org/downloads/ Guia_Para_El_Manejo_Humanitario_de_Poblaci ones_Caninas_Spanish.pdf

48. Totton SC, Wandeler AI, Ribble CS, Rosatte RC, McEwen SA. Stray dog population demographics in Joahpur, India following a population control/rabies vaccination program, 2010. Preventive Veterinary Medicine. 2011;98(2-3):51-57.

49. Ready PD. Leishmaniasis emergence in Europe. Euro Surveill [Internet]. 2010 [citado 12 jun 2012];15(10): pii=19505. Disponible en: http://www.eurosurveillance.org/ViewArticle.asp $\mathrm{x}$ ?Articleld = 19505.

50. Martín-Sánchez J, Morales-Yuste M, AcedoSánchez C, Barón S, Díaz V, Morillas-Márquez F. Canine Leishmaniasis in Southeastern Spain. Emerging Infectious Diseases. 2009;15(5):795-798.

51. Ferroglio E, Poggi M, Trisciuoglio A. Evaluation of $65 \%$ permethrin spot-on and deltamethrinimpregnated collars for canine Leishmania infantum infection prevention. Zoonoses and Public Health. 2008;55(3):145-148.

52. Camargo-Neves VLF. Utilização de coleiras impregnadas com deltametrina a $4 \%$ para o controle da leishmaniose visceral americana. En: Consulta de expertos OPS/OMS sobre leishmaniasis visceral en las Américas. Rio de Janeiro: Panaftosa-OPS; 2004.

53. Nunes CM, Pires MM, Silva KM, Assis FD, Gonçalves-Filho J, Perri SH. Relationship between dog culling and incidence of human visceral leishmaniasis in an endemic area. Veterinary Parasitology. 2010;170(1-2):131-133.

54. Marzochi MC, Fagundes A, Andrade MV, Souza MB, Madeira MF, Mouta-Confort E, Schubach AO, Marzochi KB. Visceral leishmaniasis in Rio de Janeiro, Brazil: eco-epidemiological aspects and control. Revista da Sociedade Brasileira de Medicina Tropical. 2009;42(5):570-580. 
55. Dietze R, Barros GB, Teixeira L, Harris J, Michelson K, Falqueto A, Corey R. Effect of eliminating seropositive canines on the transmission of visceral leishmaniasis in Brazil. Clinical Infectious Diseases. 1997;25(5):1240-1242.

56. Ashford DA, David JR, Freire M, David R, Sherlock I, Eulálio MC, Sampaio DP, Badaro R. Studies on control of visceral leishmaniasis: impact of dog control on canine and human visceral leishmaniasis in Jacobina, Bahia, Brazil. American Journal of Tropical Medicine \& Hygiene. 1998;59(1):53-57.

57. Quinnell RJ, Courtenay O. Transmission, reservoir hosts and control of zoonotic visceral leishmaniasis. Parasitology. 2009;136(14):19151934.

58. Argentina, el país de la región con más mascotas por habitante. Infobae [Internet]. 16 set 2011 [citado 12 jun 2012]. Disponible en: http://www.infobae.com/notas/605985Argentina-el-pais-de-la-region-con-mas-mascotaspor-habitante.html

59. Arana García E. Animales de compañía y administración local: Derecho del medio ambiente y administración local. Granada: Fundación Democracia y Gobierno Local; 2005.

60. Ingold T. What is an animal? London: OWA, Routledge; 1988.

61. Leite-Lopes JS. Sobre processos de "ambientalização" dos conflitos e os dilemas da participação. Horizontes Antropológicos. 2006;12(25):31-64.

62. Arach O. Ambientalismo, desarrollo y transnacionalidad: las protestas sociales en torno a la represa de Yacyretá. En: Jelín E. Mas allá de la nación: las múltiples escalas de los movimientos sociales. Buenos Aires: Libros del Zorzal; 2003.
63. Brites W. Políticas urbanas autoritarias: el caso de una población carenciada desplazada por grandes obras [Internet]. 4to Congreso Virtual de Antropología y Arqueología, NAyA 2004 [citado 12 jun 2012]. Disponible en: http://www.naya. org.ar/congreso2004/ponencias/walter brites.htm

64. Ferrero BG. Caza, masculinidad y ambientalismo en los montes misioneros. IX Congreso argentino de antropología social (CD-ROM). Posadas: Universidad Nacional de Misiones; 2008.

65. Crece el robo y la reventa de perros en la ciudad de Posadas. El Territorio Posadas [Internet]. 28 may 2012 [citado 12 jun 2012]. Disponible en: http://www.territoriodigital.com/nota3.aspx?c $=8407637177603105$

66. Almada CO. La información de la evolución de la LV y el inicio del proceso de investigación. En: I Encuentro de la Red de Investigación de las Leishmaniasis en Argentina. Buenos Aires: REDILA; 2010.

67. Chomel BB, Sun B. Zoonoses in the bedroom. Emerging Infectious Diseases. 2011;17(2):167-172.

68. La leishmaniasis. El Refugio [Internet]. 2007 [citado 12 jun 2012]. Disponible en: http://elrefugiodemisiones.blogspot.com.ar/2007/11/nosotros-el-refugio.html

69. Bouis S, Villalbí J, Rodríguez-Campos $M$, Perachp V, Durán J. Control de las lesiones causadas por perros: una perspectiva de salud pública. Gaceta Sanitaria. 2010;24(2):179-180.

70. Zaffaroni ER. La Pachamama y el humano. Buenos Aires: Colihue; 2012.

71. Beck U. La sociedad del riesgo: hacia una nueva modernidad. Barcelona: Paidós Ibérica; 2006.

\section{FORMA DE CITAR}

Salomón OD, Mastrángelo AV, Santini MS, Ruvinsky S, Orduna T, Sinagra A, Luna C, Riarte A, Casas N, Amiotti P. Leishmaniasis visceral: senderos que confluyen, se bifurcan. Salud Colectiva. 2012;8(Supl 1):S49-S63.

Recibido el 9 de agosto de 2012

Versión final presentada el 14 de septiembre de 2012

Aprobado el 21 de septiembre de 2012 